\title{
An attempt to shorten the inspection time of active thermography method
}

\author{
by S. Ishihara*, M. Ishikawa*, H. Nishino*, M. Koyama**, \\ H. Kasano ${ }^{\star \star \star}$, H. Hatta**** and S. Utsunomiya ${ }^{\star \star \star \star}$ \\ *Tokushima University, 2-1 Minamijousanjima-cho, Tokushima, 770-8506, Japan, m.ishikawa@tokushima-u.ac.jp \\ ${ }^{* *}$ Meisei University, 2-1-1 Hodokubo, Hino, Tokyo, 191-8506, Japan \\ *** Nihon University, Tokusada, Tamura, Koriyama, Fukushima,963-8642, Japan

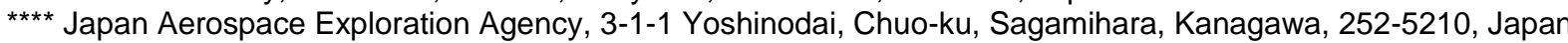

\begin{abstract}
When inspecting concrete structures or low-thermal-diffusivity materials (such as plastic materials) using active thermography method, a long inspection time is required, and this impairs the efficiency of thermographic non-destructive inspection. In this study, we focused on the use of high-frequency thermal waves which has theoretically fast heat propagation speed, and tried to shorten the inspection time. In order to verify the effectiveness of shortening inspection time, experiments for mortar specimens with an artificial defect were performed.
\end{abstract}

\section{Introduction}

Active thermographic inspection is an easy and efficient non-destructive testing method. However, it is known that the method requires a long inspection time when detecting defects in a large concrete structure or in a low-thermaldiffusivity material (such as a plastic material). For example, for detecting a defect located at a depth of several tens of millimetres in a concrete structure, several hundred or more than 1,000 seconds is required $[1,2]$. This degrade the efficiency of the active thermography method. Therefore, the aim of this study is to shorten the inspection time of active thermography method. In order to achieve the aim, we focused on using thermal waves with high frequency components.

When a surface of an object having infinite thickness changes periodically, the temperature $T$ at the depth $z$ from the surface is obtained as $[3,4,5]$

$$
T(z, t)=T_{0} \mathrm{e}^{-\frac{z}{\mu}} \cos 2 \pi\left(f t-\frac{z}{2 \pi \mu}\right)
$$

where $t$ is time, $T_{0}$ is temperature when $z=t=0$, and $f$ is frequency of the thermal wave. $\mu$ is thermal penetration depth which is obtained from thermal conductivity $k$, density $\rho$, and specific heat $C$ as

$$
\mu=\sqrt{\frac{k}{f \rho C}}=\sqrt{\frac{\alpha}{f}}
$$

here $\alpha$ denotes the thermal diffusivity. From the Eq. (1) wavelength $\lambda$ and heat propagation velocity $v$ of the thermal wave are obtained as follows:

$$
\begin{aligned}
& \lambda=2 \pi \mu \\
& v=2 \sqrt{\pi f \alpha}
\end{aligned}
$$

The Eq. (4) means that heat propagates faster when the frequency of the thermal wave is higher. Thus, in this study, we tried to use thermal waves with high frequency components, and investigated the relationship between the input heat frequency and required inspection time through experiments for mortar specimens with an artificial defect.

\section{Experiments}

A schematic of experimental setup and mortar specimens are shown in figure 1 and 2, respectively. The mortar specimens were heated by a $500 \mathrm{~W}$ halogen lamp, and surface temperature during heating was monitored by an infrared camera (A315, FLIR Systems Co.). The specimens were $70 \times 100 \times 50 \mathrm{~mm}$, and had an insertion of a 30-mm-width polystyrene foam as an artificial defect. The defect thickness was $7 \mathrm{~mm}$ and it was located at a depth of 5 or $10 \mathrm{~mm}$ below the surface. The output power of the halogen lamp was controlled by a function generator (Tektronix AFG3102) to a sine wave shape, and the modulation frequency (heating frequency) was varied as $0.01,0.03,0.05$ and $0.1 \mathrm{~Hz}$. Then, 
relationship between the heating frequency and the minimum time required to detect the defect was investigated. In this study, the phase image obtained by applying the Fourier transform to the observed temperature-time data [5,6,7] was used to determine the minimum inspection time required to detect the defect. The phase image was obtained by varying the time of temperature data used for the Fourier transform, and the minimum inspection time was determined based on the signal-to-noise ratio $(S / N)$ in the obtained phase images. Figure 3 shows an example of the phase image, and shows how to determine $S I N$. The $S$ value was determined as phase difference between the defective and non-defective area, and the $N$ value was the standard deviation of the phase in non-defective area.

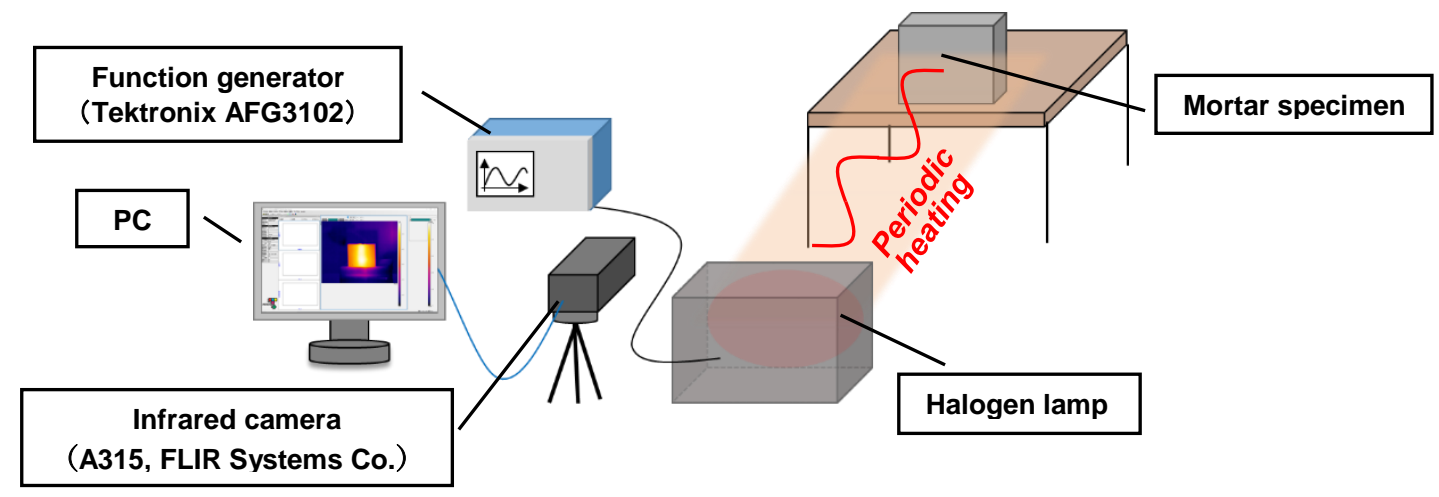

Fig. 1. Schematic of experimental setup.
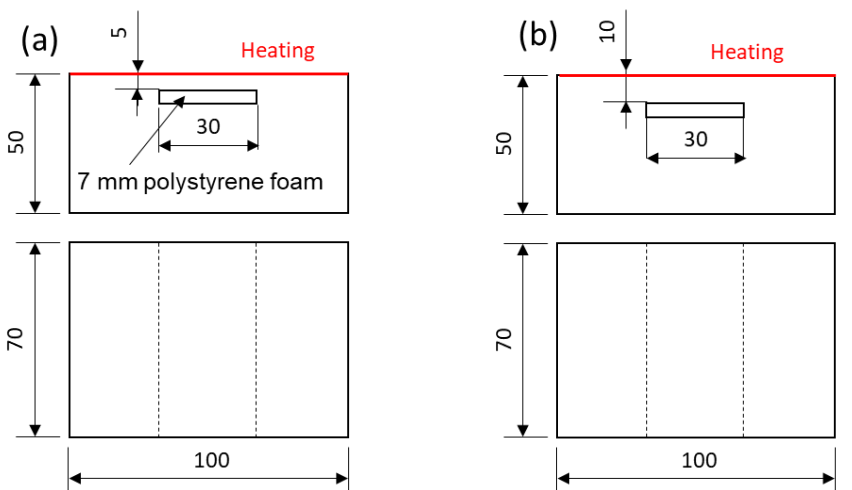

Fig. 2. Schematic of mortar specimens with a (a) 5-mm-depth defect, and (b) 10-mm-depth defect.

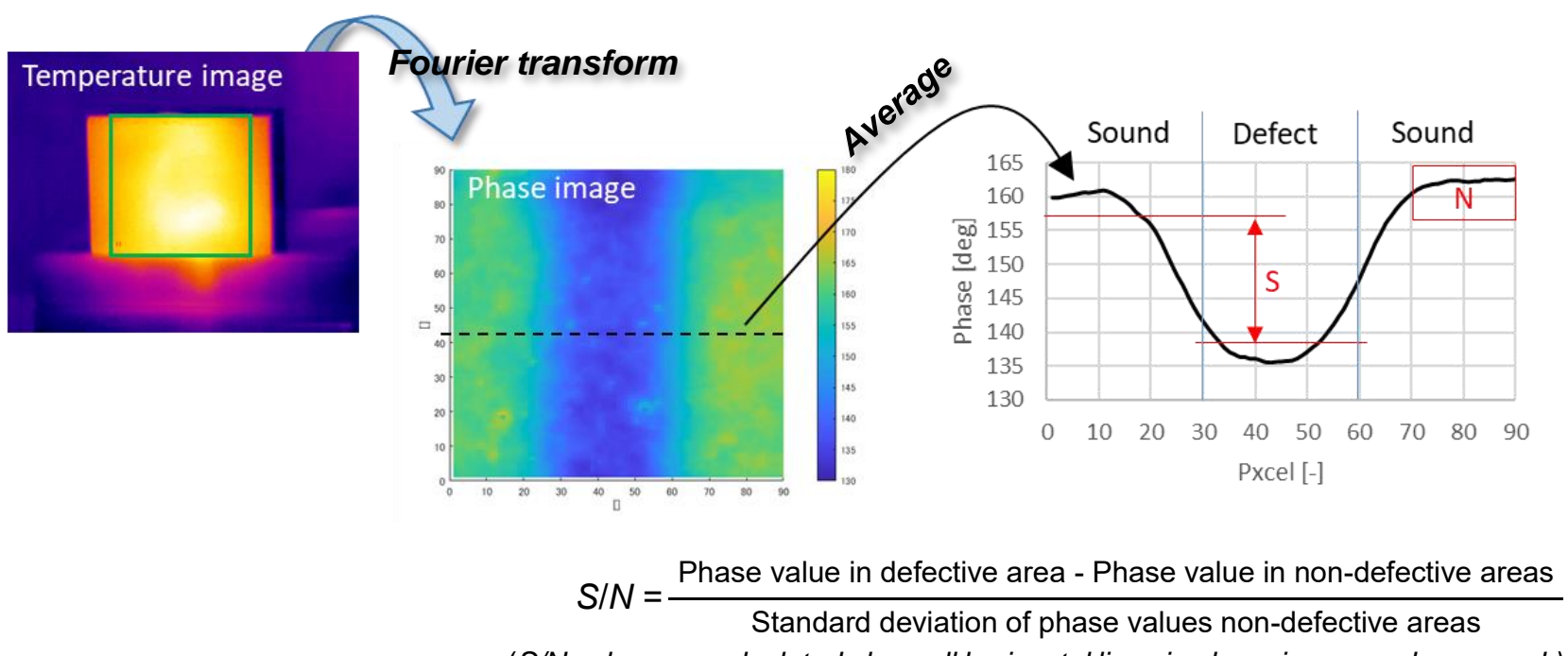

( $S / N$ value was calculated along all horizontal lines in phase image and averaged.)

Fig. 3. How to determine the signal-to-noise ratio $(S / N)$ from the obtained phase images. 


\section{Results and Discussion}

\subsection{Relationship between $S / N$ and inspection time}

Figure 4 and 5 show the phase images of the specimen with the $5-\mathrm{mm}$ - and 10-mm-depth defect, respectively, obtained when the heating frequency is 0.01 and $0.1 \mathrm{~Hz}$. It is found from the images that, although the artificial defect could not be detecd when the inspection time is short, it could be gradually detected with time. Figure 6 shows the relationships between the inspection time and $S / N$. The $S / N$ values increase with increasing the time, and its increment speed varies depending on the heating frequency. By comparing the phase images with the $S / N$ values, the time when $S / N$ reaches 4 was determined as the minimum inspection time required to detect the defect in this study (figure 4(b) and (e), figure 5(b) and $(\mathrm{e})$ are the phase image when $S / N=4)$. In figure 7 , the relationship between the time when the $S / N=4$ and the heating frequency is presented. These results show that the minimum inspection time shows a decrease tendency with the heating frequency. This means the required inspection time becomes shorter by increasing the heating frequency.

\subsection{Investigation of frequency components and attenuation of input heat}

To examine the reason why the inspection time was shortened by increasing the heating frequency, the frequency components in the input heat was investigated. The observed temperature-time data was Fourier transformed, and then obtained spectrum amplitude is displayed as functions of frequency and inspection time in figure 8 (the vertical and horizontal axis shows the frequency and inspection time, respectively, and the color shows the spectrum amplitude). In the result of $0.01 \mathrm{~Hz}$ heating (figure 8(a)), almost no spectrum is observed when the inspection time is less than $20 \mathrm{~s}$. In contrast, a wider band spectrum is input in shorter time when the heating frequency is higher. These results imply that, by increasing the heating frequency, thermal waves with higher frequency components, which has faster heat propagation velocity, is input and propagate between the surface and subsurface defect in shorter time.

(a)

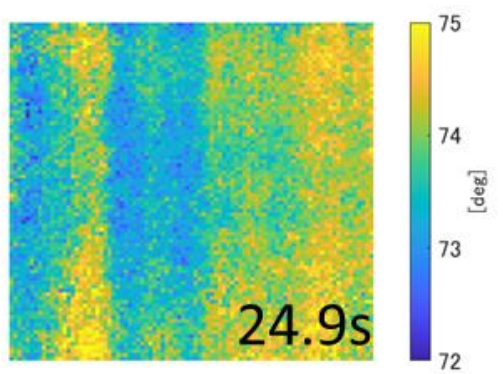

(d)

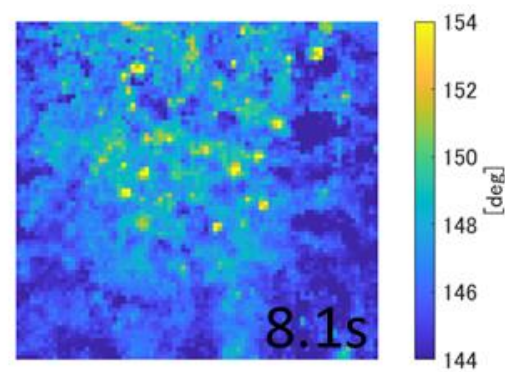

(b)

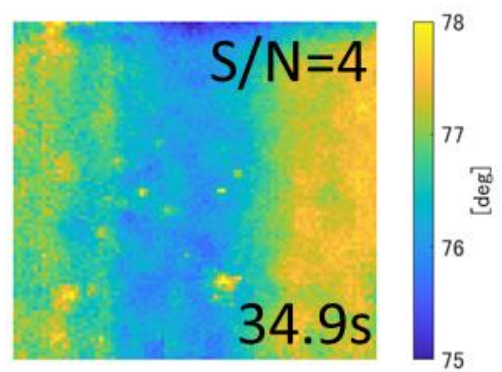

(e)

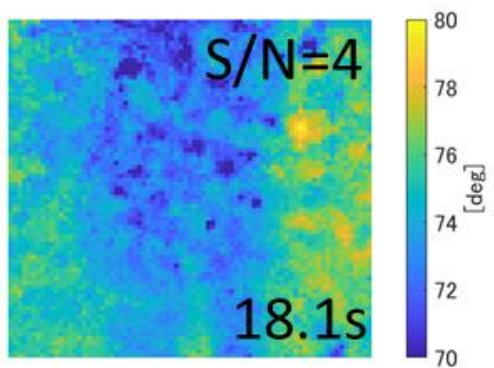

(c)

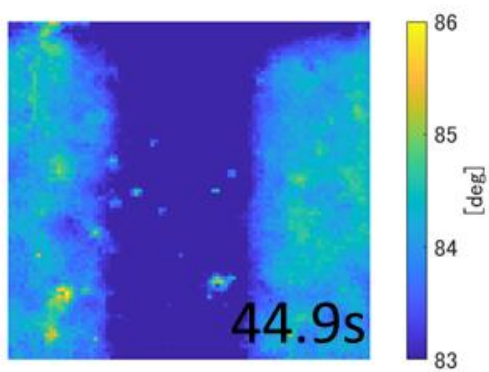

(f)

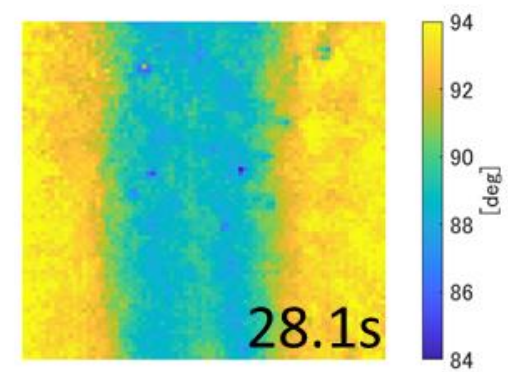

Fig. 4. Experimentally obtained phase images of the specimen with 5-mm-depth defect when the heating frequency is (a)-(c) $0.01 \mathrm{~Hz}$ and (d)-(f) $0.1 \mathrm{~Hz}$. The inspection time is (a) $24.9 \mathrm{~s}$, (b) $34.9 \mathrm{~s}(\mathrm{~S} / \mathrm{N}=4)$, (c) $44.9 \mathrm{~s}$, (d) $8.1 \mathrm{~s},(e) 18.1 \mathrm{~s}(\mathrm{~S} / \mathrm{N}=4)$, and (f) $28.1 \mathrm{~s}$. 
(a)

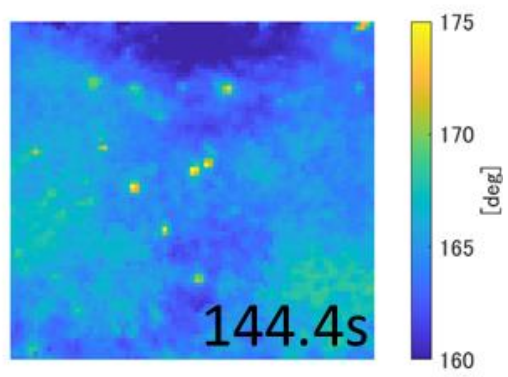

(d)

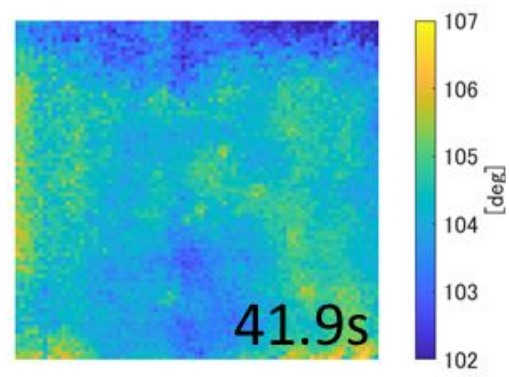

(b)

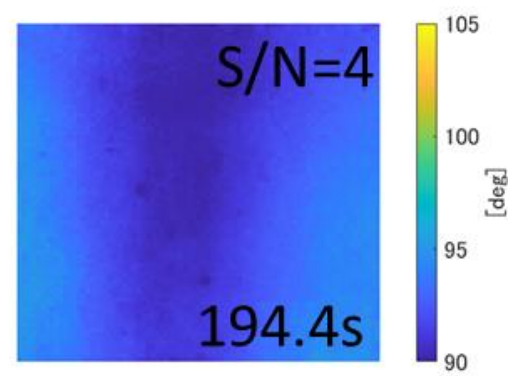

(e)

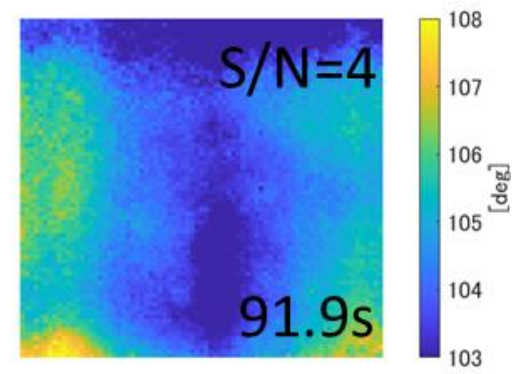

(c)

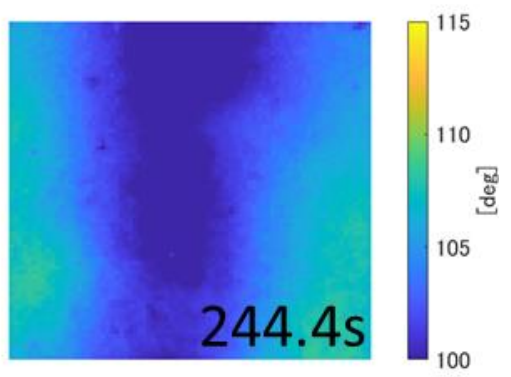

(f)

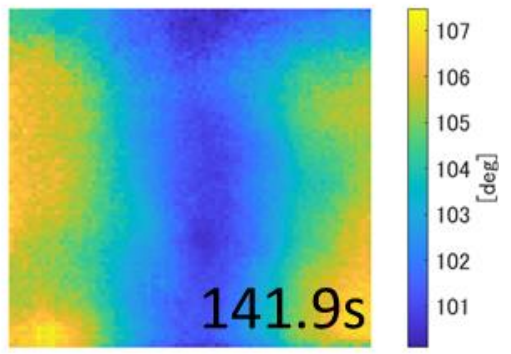

Fig. 5. Experimentally obtained phase images of the specimen with 10-mm-depth defect when the heating frequency is (a)-(c) $0.01 \mathrm{~Hz}$ and (d)-(f) $0.1 \mathrm{~Hz}$. The inspection time is (a) $144.4 \mathrm{~s}$, (b) $194.4 \mathrm{~s}(\mathrm{~S} / \mathrm{N}=4)$, (c) $244.4 \mathrm{~s}$, (d) $41.9 \mathrm{~s}$, (e) $91.9 \mathrm{~s}(\mathrm{~S} / \mathrm{N}=4)$, and (f) $141.9 \mathrm{~s}$.

(a)

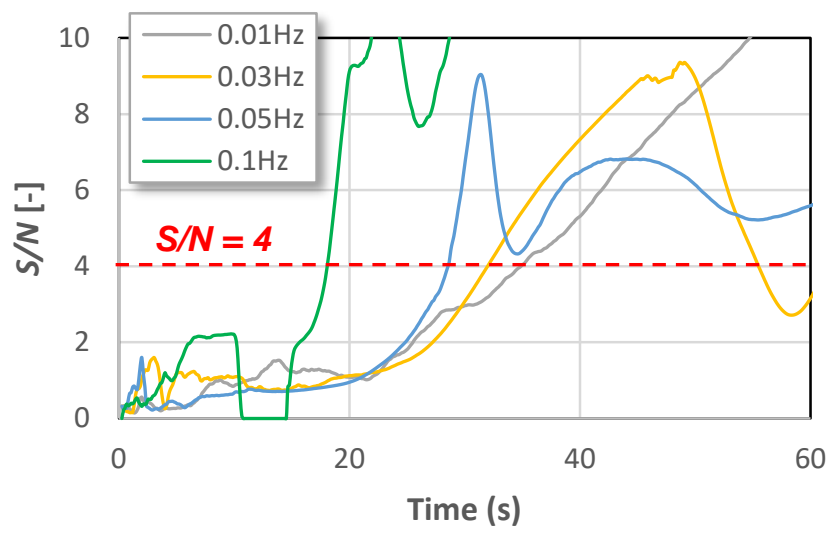

(b)

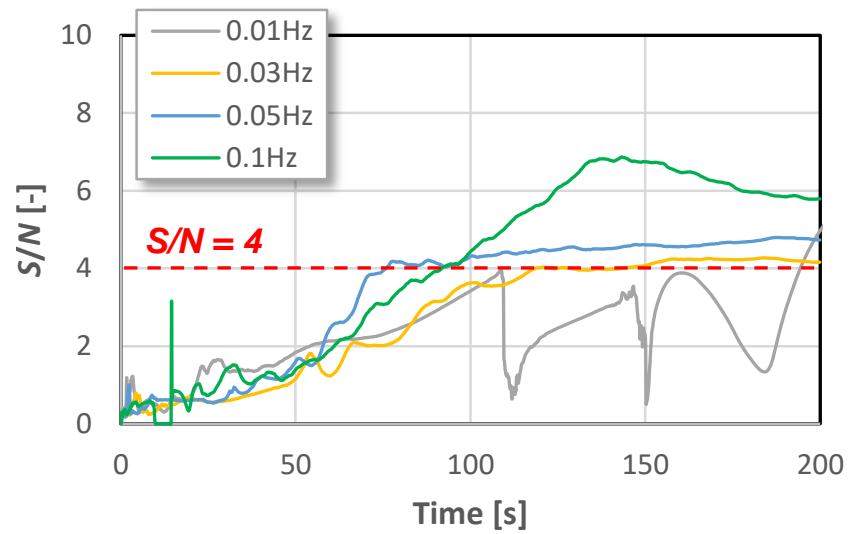

Fig. 6. Relationship between the inspection time and $\mathrm{S} / \mathrm{N}$ in phase image obtained when detecting (a) 5-mmdepth defect, and (b) 10-mm-depth defect.

It should be noted in the experimental result of the specimen with a 10-mm-depth defect (figure 7(b)) that the minimum inspection time does not decrease when the heating frequency increases from 0.05 to $0.1 \mathrm{~Hz}$. This should be because of the effect of thermal wave attenuation. From Eq. (1) and (2), it is found that the amplitude of the thermal wave decreases exponentially with increasing the frequency based on the attenuation coefficient of. Figure 9 shows a theoretically calculated relative amplitude of a thermal wave that reflected from 5- and 10-mm-depth defect in a mortar (amplitude ratio of reflected to input thermal wave) as a function of thermal wave frequency. This figure shows, for example, the amplitude of the thermal wave with the frequency of $0.1 \mathrm{~Hz}$ decreases to approximately $10^{-7}$ times of the input amplitude when it propagates round-trip distance to the 10-mm-depth defect. This means the thermal wave with such high frequency components hardly returns to the observation surface, and it does not contribute to defect detection. 

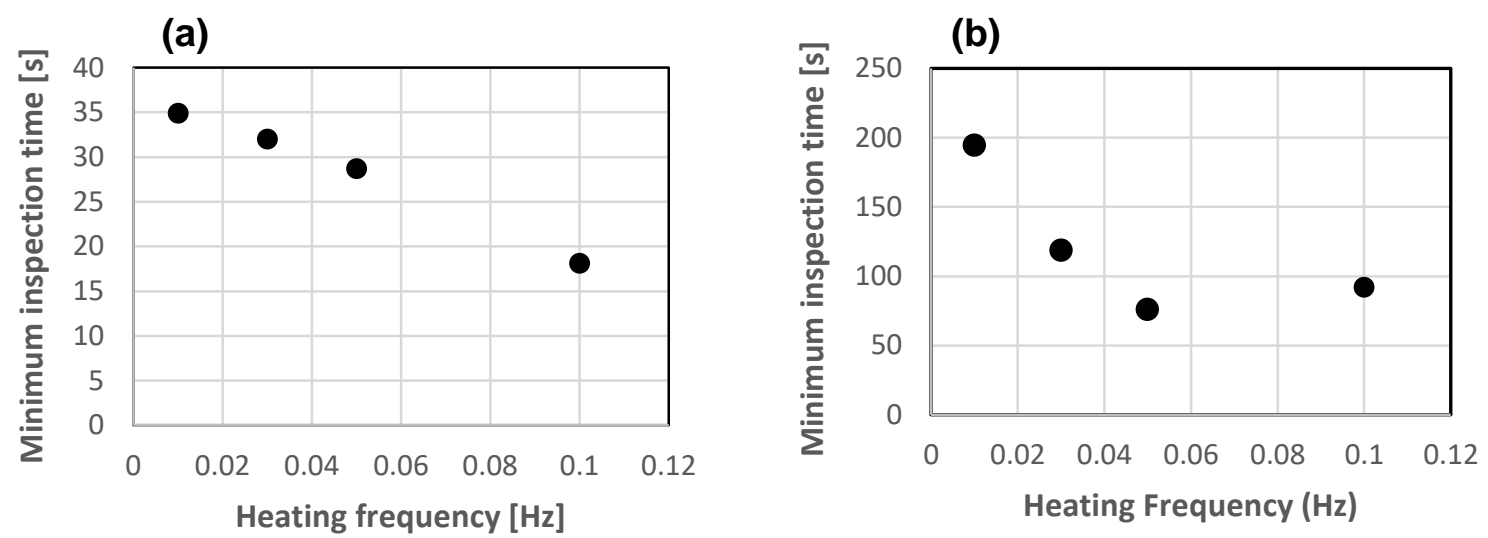

Fig. 7. Relationship between the minimum inspection time (the time when $S / N=4$ ) and heating frequency when detecting (a) 5-mm-depth defect,and (b) 10-mm-depth defect.

(a)

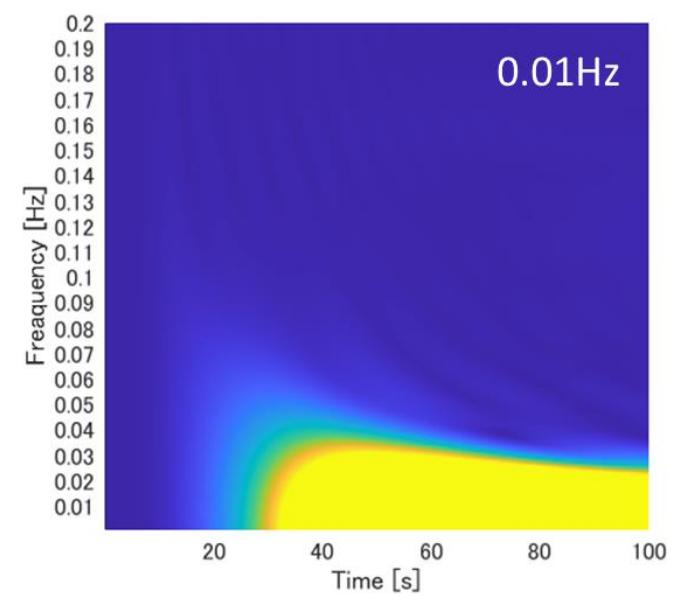

(c)

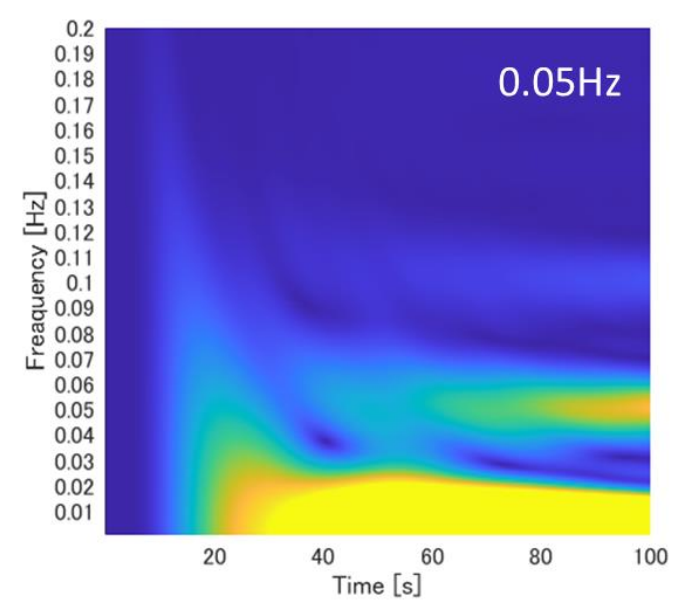

(b)
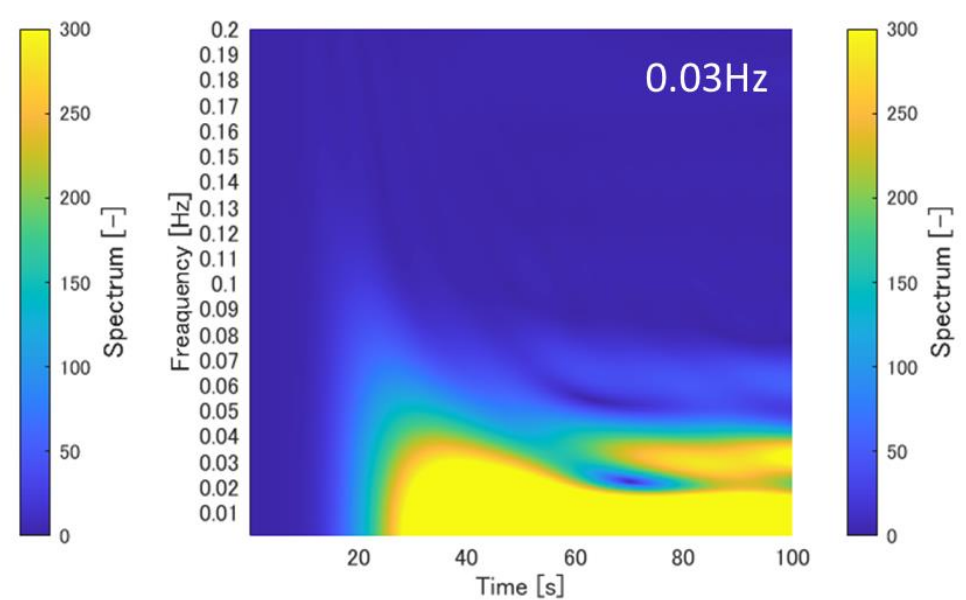

(d)
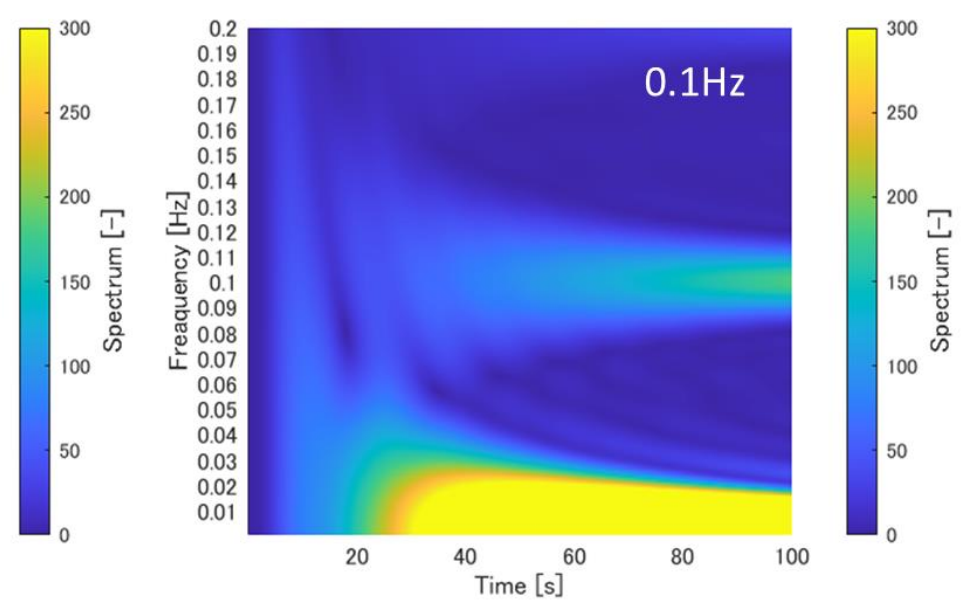

Fig. 8. Spectrum amplitude of the experimentally input thermal wave as functions of frequency and inspection time obtained when the heating frequency is (a) $0.01 \mathrm{~Hz}$, (b) $0.03 \mathrm{~Hz}$, (c) $0.05 \mathrm{~Hz}$, and (d) $0.1 \mathrm{~Hz}$. 


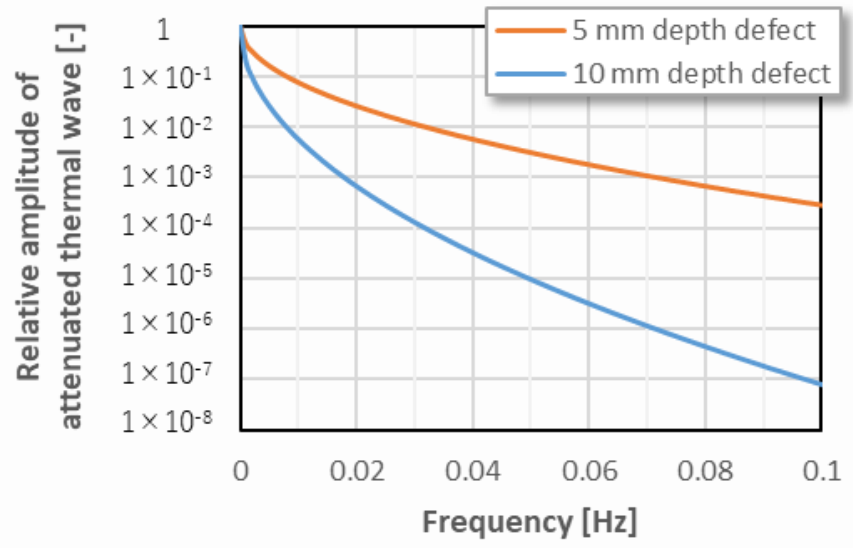

Fig. 9. Relative temperature amplitude of thermal wave after propagation of round-trip distance to a 5-and 10-mm-depth defect as a function of frequency.

\section{Conclusions}

In order to shorten the inspection time of active thermographic non-destructive testing, the effect of varying the heating frequency of periodic heating and inputting higher frequency thermal waves that has theoretically higher heat propagation velocity were examined. Results for mortar specimens showed that the inspection time required to detect the defect decreased with increasing the heating frequency; this should be because the thermal waves with higher frequency components was input in shorter time by increasing the heating frequency. Therefore, these results suggest inputting high frequency thermal waves is an effective way to shorten the inspection time. However, in our experiments, the reduction of inspection time was not observed when detecting 10-mm-depth defect using the heating frequency of $0.1 \mathrm{~Hz}$. This should be because of the large attenuation during heat propagation, and shows that the time reduction effect could not be obtained when the thermal wave frequency is too high to detect it.

\section{Acknowledgements} 17 K01296

This work was supported by Japan Society for the Promotion of Science (JSPS) KAKENHI Grant Numbers

\section{REFERENCES}

[1] Cotič, P., Kolarič, D., Bosiljkov, V. B., Bosiljkov, V., Jagličić, Z., Determination of the applicability and limits of void and delamination detection in concrete structures using infrared thermography. NDT \& E Int. - Vol. 74, pp. 8793, 2015.

[2] Huh, J., Tran, Q.H., Lee, J.H., Han, D., Ahn, J.H., Yim, S., Experimental Study on Detection of Deterioration in Concrete Using Infrared Thermography Technique. Advances in Materials Science and Engineering, 2016.

[3] Almond D.P., Patel P., "Photothermal Science and Techniques", Chapman and Hall, London (1996).

[4] Maldague X., "Theory and practice of infrared technology for nondestructive testing", John Wiley \& Sons, New York (2001).

[5] Maldague X., Galmiche F., Ziadi A., Advances in pulse phase thermography. Infrared Phys. Technol. - Vol. 43, pp.175-181, 2002.

[6] Maldague X., Marinetti S., Pulse phase infrared thermography, J. appl. Phys. - Vol. 79, no 5, pp. 2694-2698, 1996.

[7] Ishikawa M., Hatta H., Habuka Y., Fukui R., Utsunomiya S., Detecting deeper defects using pulse phase thermography. Infrared Phys. Technol. - Vol. 57, pp. 42-49, 2013. 